\title{
A non-mammalian in vivo model for cellular and molecular analysis of glucose-mediated thickening of basement membranes
}

\author{
X.Zhang ${ }^{1}$, J.K. Huff ${ }^{1}$, B. G. Hudson ${ }^{2}$ and M. P. Sarras, Jr. ${ }^{1}$ \\ 1 Department of Anatomy and Cell Biology and ${ }^{2}$ Department of Biochemistry and Molecular Biology, University of Kansas Medical \\ Center, Kansas City, Kansas, USA
}

\begin{abstract}
Summary. An increase of basement membrane thickness in renal glomeruli, blood vessels, and other tissues is a consistent pathological observation in individuals with diabetes mellitus. Although a number of pathological complications of the disease are thought to result from this structural abnormality in basement membranes, the mechanism(s) responsible for this glucose-mediated process remain unknown. The current study was designed to develop a non-mammalian in vivo epithelial/basement membrane model which would facilitate detailed analysis of the cellular and molecular processes which lead to thickening of basement membrane under hyperglycaemic conditions. The system developed utilizes the Cnidarian, Hydra vulgaris. Hydra lends itself to such studies because of (1) its simplified body structure which is composed of an epithelial bilayer with an intervening basement membrane (mesoglea) and (2) its extensive regenerative capacity which allows cell pellets (Hydra cell aggre-
\end{abstract}

gates), formed from isolated Hydra cells, to develop into adult Hydra within 72-96 h. This process involves reformation of an epithelial bilayer and de novo biosynthesis of a basement membrane. Our studies indicate that exposure of developing Hydra cell aggregates to levels of D-glucose which mimic that observed in the human diabetic patient $(15 \mathrm{mmol} / \mathrm{l})$ induces a doubling of Hydra basement membrane thickness within $72-96$ h of pellet formation. The same results were obtained using $15 \mathrm{mmol} / \mathrm{l} \mathrm{D}$-Ribose which is a highly efficient glycating agent. The data presented support the use of the Hydra cell aggregate system as a potentially powerful non-mammalian in vivo model to investigate the cellular and molecular mechanism(s) underlying glucosemediated basement membrane thickening.

Key words: Diabetes, basement membrane, hydra, hyperglycaemia, animal models.
Thickening of basement membrane in renal glomeruli, blood vessels, and other tissues is a common pathological observation in individuals with diabetes mellitus $[1,2]$. While various pathological complications of the disease are thought to result from this structural abnormality in basement membranes, the mechanism(s) responsible for this glucose-mediated process remain unknown $[1,2]$. Investigations regarding this problem have been conducted utilizing human tissues and a number of different animal models [2]. One problem associated with the use of animal models is the time required for structural changes to occur in basement membranes. This period varies from four to eight months for spontaneous [3] and streptozotocin (STZ)-induced [4] diabetic rats and can be as long as six years for pancreactomized monkeys [5]. This protracted time frame introduces secondary factors such as age and diet which complicate analysis of the cellular mechanisms directly involved in basement membrane thickening. These problems could be alleviated by a simplified in vitro or in vivo cellular model in which basement membrane thickening occurred more rapidly after exposure to hyperglycaemic conditions.
Fig. 1a-f. Hydra cell aggregate development under control and elevated D-glucose or D-ribose conditions. As viewed by scanning electron microscopy $(\mathbf{a}, \mathbf{b})$, Hydra cell aggregates develop into a fluid-filled cyst by $24 \mathrm{~h}$ after cell pellet formation. As shown in a, cysts are formed by an epithelial bilayer. The arrows in $\mathbf{a}$ indicate the apical border of each adjacent epithelial layer. At higher magnification (b), the organization of this epithelial bilayer as an ectoderm $(\mathrm{Ec})$ and endoderm (En) layer with an intervening basement membrane (mesoglea) (indicated by arrows) is shown. Ultrastructural analysis of basement membrane formation at $96 \mathrm{~h}$ of Hydra cell aggregate development under control (c) (minus D-glucose) and experimental (d) $(15 \mathrm{mmol} / 1 \mathrm{D}$-glucose) conditions indicated an increase in basement membrane thickness with elevated glucose concentrations (d). The arrowheads shown in $1 \mathrm{c}$ and $1 \mathrm{~d}$ mark the outer margins of basement membrane. The bottom left graph (e) depicts quantitation of unit Hydra basement membrane area (reflecting thickness as measured in this analysis) under control and elevated $(15 \mathrm{mmol} / \mathrm{l}) \mathrm{D}$-glucose or D-ribose $\square$ conditions at $96 \mathrm{~h}$ of development. The bottom right graph (f) depicts unit Hydra basement membrane area under normal $\square$ and elevated D-ribose (100 mmol/l) conditions at $96 \mathrm{~h}, 7$ days, and 12 days of development. Standard errors are indicated on the top of each bar with $n=4$. Control and experimental groups were statistically different at $p<0.05$. The control groups shown in $1 \mathrm{f}$ were not statistically different at $p<0.05$. Magnifications: $1 \mathrm{a}, 160 \mathrm{X} ; 1 \mathrm{~b}, 835 \mathrm{X} ; 1 \mathrm{c}$ and $1 \mathrm{~d}$, $13,500 \mathrm{X}$ 

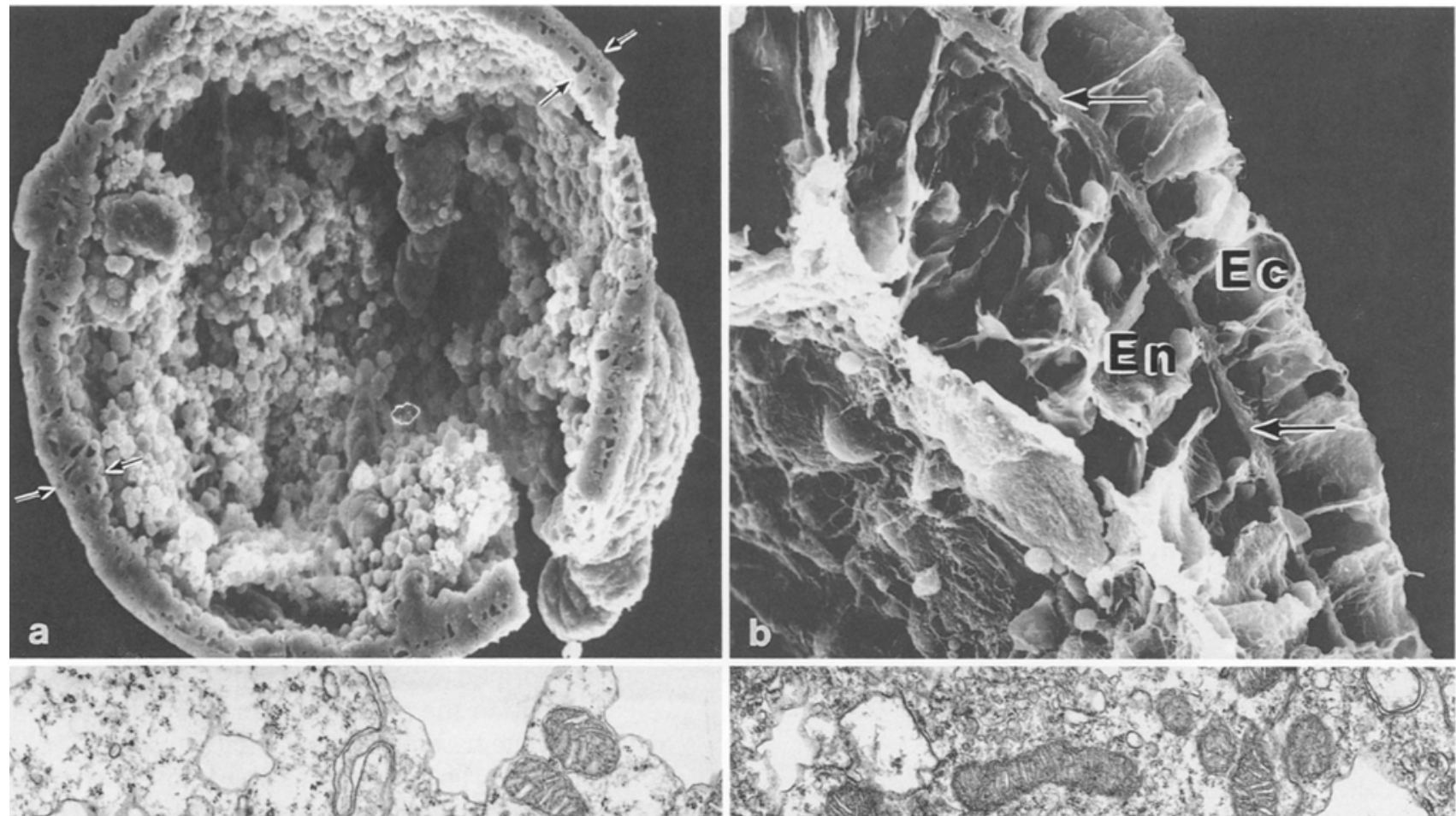

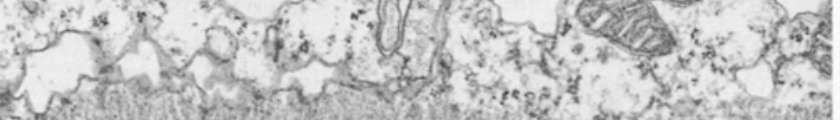

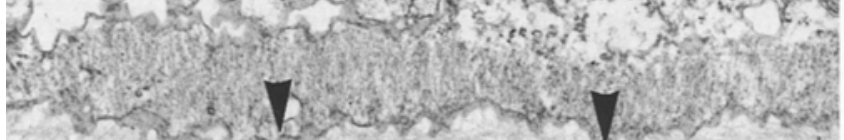

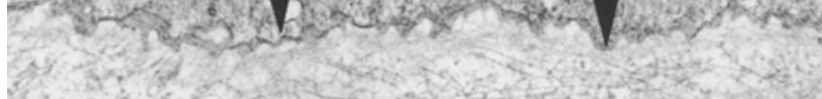

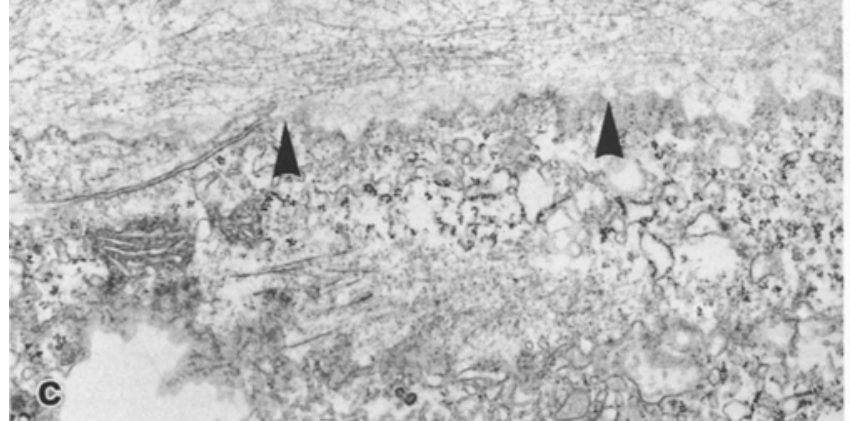

C

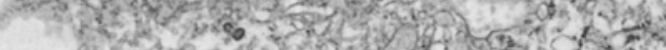
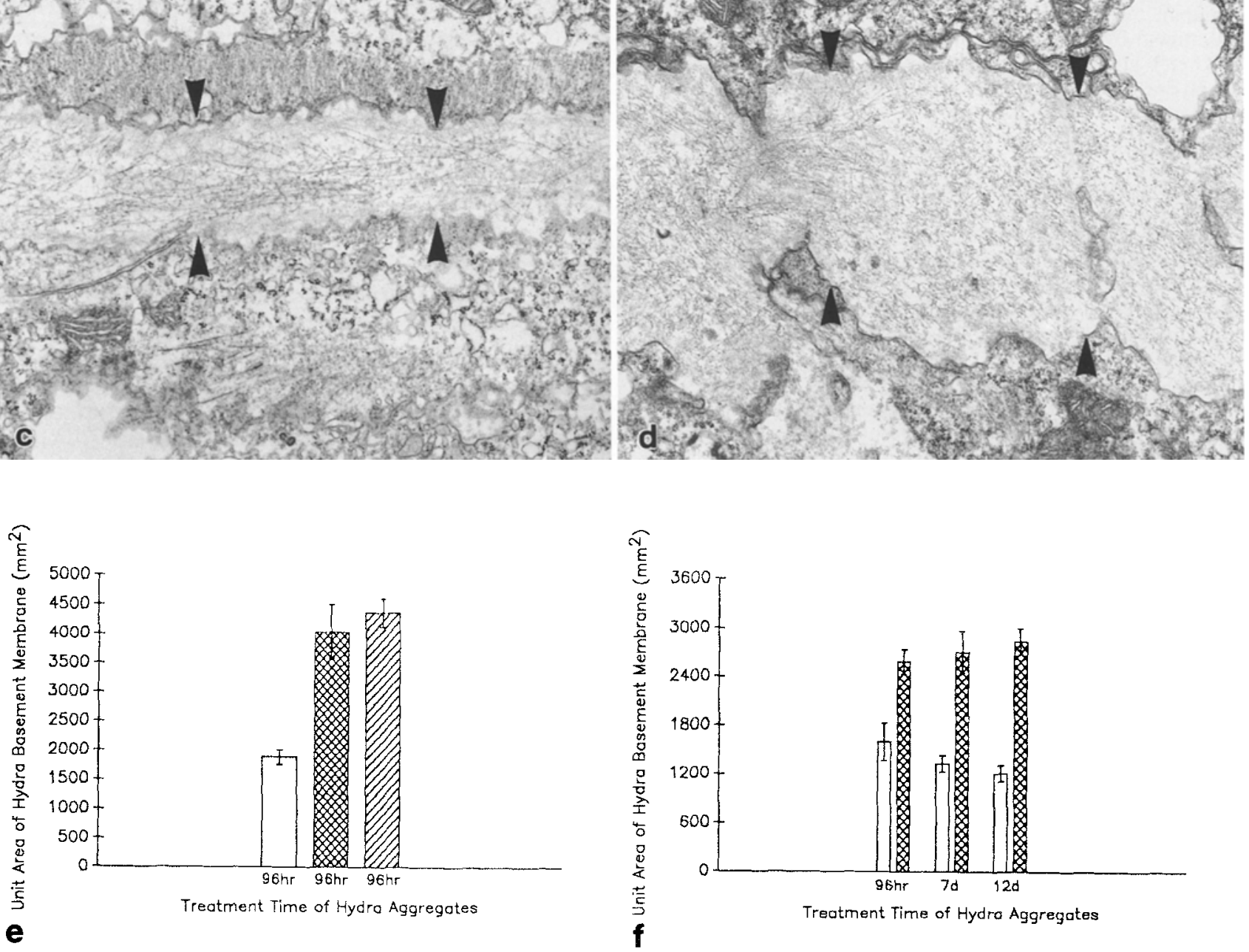
In an attempt to circumvent the problems associated with diabetic animal models, we have investigated the use of Hydra vulgaris as an alternative in vivo cellular model. Hydra have distinct advantages in studies related to the formation and maintenance of basement membranes. Structurally, Hydra is reduced to an epithelial bilayer with an intervening basement membrane which has a composition similar to that observed in mammals [6]. In addition, the organism has an extensive regenerative capacity which allows cell pellets (Hydra cell aggregates) formed from isolated Hydra cells to reform into adult Hydra within $72-96 \mathrm{~h}$. This morphogenetic process involves reformation of an epithelial bilayer and subsequent de novo biosynthesis of a basement membrane. The objective of the present study was to determine if elevated glucose levels induce thickening of Hydra basement membrane during development of Hydra cell aggregates.

\section{Materials and methods}

Hydra vulgaris (formally Hydra attenuata) colonies were maintained in specially designed aquariums using a culture medium containing: $1 \mathrm{mmol} / \mathrm{l} \mathrm{CaCl}, 1 \mathrm{mmol} / 1 \quad \mathrm{NaHCO}_{3}, 0.25 \mathrm{mmol} / \mathrm{l} \mathrm{MgCl}_{2}$, and $0.01 \mathrm{mmol} / \mathrm{l}$ EDTA. Hydra cell aggregates were made as described by Gierer et al. [7] with the following minor modifications: (1) 500 Hydra were minced prior to dissociation; (2) dissociated cells were further filtered through a $53 \mu \mathrm{m}$ Nytex screen; and (3) 500,000 cells were used for formation of each Hydra cell aggregate pellet. Once formed, pellets were immediately transferred into sterilized Hydra medium and $0.005 \%$ rifampicin. At $24 \mathrm{~h}$ of development, experimental Hydra cell aggregates were transferred to Hydra medium containing varying concentrations of $\mathrm{D}$-glucose or D-ribose. Experiments were terminated at 96 h, 7 days, and 12 days after pellet formation. At the end of each experiment, Hydra cell aggregates were fixed and processed into Spurr's resin and stained ultrathin sections were examined using a JEOL $100 \mathrm{~S}$ transmission electron microscope.

Blocks were trimmed and faced to ensure that cross sections were obtained from the Hydra cell aggregates. Given the structure of Hydra (see Fig. 1 a, b), cross sectional planes could be identified by trimming and facing specimens until an epithelial bilayer with an intervening basement membrane was observed in thick sections. Oblique sectioning would result in the appearance of multiple epithelial layers. When such oblique sections were observed, trimming and facing was continued until a cross sectional plane was obtained. Cross sectional planes for control and experimental specimens were therefore within the variance of the width of an epithelial cell of the ectodermal and endodermal layer. At the ultrastructural level, Hydra basement membranes were easily identified using morphological criteria and all electron micrographs were taken at a magnification of $8,000 \mathrm{X}$. Once a basement membrane was identified, all regions along its length were photographed. This resulted in a minimum of 30 micrographs being taken for each specimen. A minimum of two specimens were examined for each control and experimental group and each experiment was repeated at least twice. This procedure prevented a biased selection of particularly thick or thin regions. In addition, all experiments were conducted in parallel by two investigators to determine consistency of results. A standardized enlargement was used so that a basement membrane length of $9 \mathrm{~cm}$ was measured on each photomicrograph. This meant that all measurements were of a standard unit length so that increases in mean volume values represented increases in basement membrane thickness. For each control and experimental specimen, basement membrane area on each photomicrograph (each photomicrograph represented adjacent areas of the basement membrane) was deter- mined using a Sigma Scan (Jandel Scientific, Corte Madera, Calif., USA) morphometric program installed on a Zenith $159 \mathrm{PC}$ with a SummaSketch digitizer pad. Data were expressed in square millimeters as measured directly from the photomicrographs. For the magnifications used in these experiments, actual in situ square micrometers can be determined by multiplying square millimeters by a factor of 0.0055 .

\section{Statistical analysis}

Control and experimental groups were statistically compared using the Student's ' $t$ ' test. When groups of more than two were compared, analysis of variance was used. For all data points shown in Figures $1 \mathrm{e}$ and $\mathrm{f}$, a minimum of four $(n=4)$ separate groups were utilized.

\section{Results}

As described by Gierer et al. [7], Hydra cell aggregate pellets develop into a cyst by $24 \mathrm{~h}$. As shown in Figure $1 \mathrm{a}$, cysts are formed by an epithelium bilayer. Once Hydra cells sort within the pellet to form an epithelium bilayer, an intervening basement membrane (mesoglea) begins to be formed (Fig. 1 b). Representative photomicrographs of control and experimental ( $15 \mathrm{mmol} / \mathrm{l}$ glucose) Hydra cell aggregates taken $96 \mathrm{~h}$ after pellet formation are shown in Figures $1 \mathrm{c}$ and $1 \mathrm{~d}$, respectively. While the ultrastructure of basement membrane appears similar between control and experimental specimens, the increased thickness of the glucose-treated specimen is apparent. As shown in the bottom left graph (Fig. 1e), exposure of Hydra cell aggregates to $15 \mathrm{mmol} / \mathrm{l}$ glucose or $15 \mathrm{mmol} / \mathrm{l}$ ribose resulted in a doubling of basement membrane area within $96 \mathrm{~h}$ of pellet formation ( $72 \mathrm{~h}$ actual exposure to carbohydrate). This same result was obtained using carbohydrate concentrations as high as $100 \mathrm{mmol} / \mathrm{l}$ (Fig. 4f, data shown for ribose only). Exposure to the highest concentrations of carbohydrate for up to 12 days did not cause any additional thickening of Hydra basement membrane over what was observed after 72-96 h of treatment (Fig. 1f). Although a downward trend in basement membrane thickness appears to occur between $96 \mathrm{~h}$ and 12 days in the control groups (Fig.1f), there was no statistical difference between these groups at $p<0.05$. In all experiments; however, control and experimental groups were statistically different at $p<0.05$.

\section{Discussion}

Diabetes mellitus is a major health problem with severe secondary complications involving retinopathy, neuropathy, accelerated atherosclerosis, and nephropathy. Although glucose-mediated thickening of basement membrane has been linked to these secondary complications, the mechanism(s) triggering abnormalities in basement membrane have not been determined. To circumvent problems associated with the use of mammalian animal models of diabetes in elucidation of the precise cellular and molecular mechanisms involved in basement membrane thickening, the present study was initiated. Our 
data indicate that Hydra cell aggregates will respond to elevated glucose levels by doubling their basement membrane thickness within $96 \mathrm{~h}$ of pellet formation. The concentrations of glucose used in this study $(15 \mathrm{mmol} / 1)$ mimic those observed in individuals with diabetes and are one half the concentrations typically seen in animal models such as the STZ-treated rat [4]. This observation coupled with the fact that Hydra are structurally reduced to an epithelial bilayer with an intervening basement membrane (e.g. structurally analogous to the glomerular filtration unit) makes this non-mammalian in vivo system particularly useful in studies related to analysis of the cellular and molecular mechanisms underlying glucosemediated basement membrane thickening. The system offers a wide variety of experimental approaches not practical in mammalian animal models such as: (1) cell transfection studies using isolated Hydra cells prior to pellet formation; (2) the ability to initiate co-ordinated development of up to 500 Hydra cell aggregates to allow subsequent analysis of transcriptional and translational events associated with the synthesis of basement membrane components within the epithelium; and (3) as previously reported [6], the ability to isolate large quantities of purified Hydra basement membrane for detailed biochemical analysis of its components.

Although phylogenetically far removed from mammals, Hydra basement membrane has been shown to have a composition similar to that of mammals [6]. For example Hydra basement membrane has been reported to contain type IV collagen, laminin, fibronectin, and heparan sulfate proteoglycan [6]. In addition, immunological and biochemical analyses indicate some structural similarities between the matrix components existing in Hydra and mammalian basement membranes [6]. Taken in total, these observations support the use of Hydra as a model system to study the developmental relationship between epithelial cells and the biogenesis and maintenance of basement membrane under normal and hyperglycaemic conditions.

With these facts in mind, the Hydra cell aggregate system seems well suited to evaluate current hypotheses regarding the cellular and molecular mechanism(s) underlying glucose-mediated basement membrane thickening. Briefly stated, current hypotheses attribute basement membrane thickening in diabetes to: (1) increased rates of synthesis and/or secretion of basement membrane components [8], (2) decreased turnover or degradation of basement membrane components [9], and/or (3) extracellular structural modifications of basement membrane components resulting from extracellular non-enzymatic glycation [8]. While the current study does not directly ad- dress these hypotheses, it is interesting to note that ribose, which is a rapid glycating agent [10], has effects similar to that seen with glucose indicating a possible link between non-enzymatic glycation of matrix components and basement membrane thickening in Hydra. Further experiments are in progress to evaluate these various mechanisms using the Hydra cell aggregate system.

Acknowledgements. This work was supported by NIH grants RR 06500, DK 38219 (MPS) and DK 18381 (BGH); funds from the Juvenile Diabetes Foundation International (MPS): and funds from the University of Kansas Medical Center BRSG program awarded to MPS.

\section{References}

1. Martinez-Hernandez A, Amenta S (1983) The basement membrane in pathology. Lab Invest 48: 656-677

2. Mauer SM, Steffes MW, Brown DM (1981) The kidney in diabetes. Am J Med 70: 603-612

3. Cohen AJ, McGill PD, Rosseti RG, Guberski DL, Like AA (1987) Glomerulopathy in spontaneously diabetic rat. Diabetes 36: 944-951

4. Fluckiger W, Perrin IV, Rossi GL (1984) Morphometric studies on retinal microangiopathy and myocardiopathy in hypertensive rats (SHR) with induced diabetes Virchows Arch [Cell Pathol] 47: 79-94

5. Jones CW, West MS, Hong DT, Jonasson O (1984) Peripheral glomerular basement membrane thickness in the normal and diabetic monkey. Lab Invest 51: 193-198

6. Sarras MP, Jr, Madden ME, Meador DT, Hudson BG (1988) Isolation, characterization, and functional analysis of Hydra basement membrane (mesoglea). J Cell Biol 107: 597 a (Abstract)

7. Gierer A, Berking S, Bode H, David CN, Flick K, Hansmann G, Schaller H, Trenkner E (1972) Regeneration of hydra from reaggregated cells. Nature New Biol 239: 98-101

8. Frank RN (1984) On the pathogenesis of diabetic retinopathy. Opthalmology 91: 626-634

9. Romen W, Lange HW, Hempel K, Heck Th (1981) Studies on collagen metabolism in rats. II turnover and amino acid composition of the collagen of glomerular basement membrane in diabetes mellitus. Virchows Arch [Cell Pathol] 36: 313-320

10. Tanaka S, Avigad G, Eikenberry EF, Brodsky B (1988) Isolation and partial characterization of collagen chains dimerized by sugar-derived cross-links. J Biol Chem 263: 17650-17657

Received: 15 June 1990

and in revised form: 28 August 1990

Dr. M. P. Sarras Jr.

Department of Anatomy and Cell Biology

University of Kansas Medical Center

$39^{\text {th }}$ and Rainbow Blvd.

Kansas City, Kansas, 66103

USA 